\title{
AIRPORT TRAFFIC CONFLICT DETECTION AND RESOLUTION ALGORITHM EVALUATION
}

\author{
Denise R. Jones, Ryan C. Chartrand, Sara R. Wilson, and Sean A. Commo \\ National Aeronautics and Space Administration, Hampton, VA \\ Sharon D. Otero and Glover D. Barker, Lockheed Martin, Hampton, VA
}

\begin{abstract}
A conflict detection and resolution (CD\&R) concept for the terminal maneuvering area (TMA) was evaluated in a fast-time batch simulation study at the National Aeronautics and Space Administration (NASA) Langley Research Center. The CD\&R concept is being designed to enhance surface situation awareness and provide cockpit alerts of potential conflicts during runway, taxi, and low altitude air-to-air operations. The purpose of the study was to evaluate the performance of aircraftbased CD\&R algorithms in the TMA, as a function of surveillance accuracy. This paper gives an overview of the CD\&R concept, simulation study, and results.
\end{abstract}

\section{Introduction}

The Next Generation Air Transportation System (NextGen) concept for the year 2025 and beyond envisions the movement of large numbers of people and goods in a safe, efficient, and reliable manner [1]. NextGen will remove many of the constraints in the current air transportation system, support a wider range of operations, and provide an overall system capacity up to three times that of current operating levels. Emerging NextGen operational concepts [2], such as four-dimensional trajectory based airborne and surface operations, equivalent visual operations, and super density arrival and departure operations, require a different approach to air traffic management and as a result, a dramatic shift in the tasks, roles, and responsibilities for the flight deck and air traffic control (ATC) to ensure a safe, sustainable air transportation system.

The National Aeronautics and Space Administration (NASA) is conducting research to develop technologies, data, and guidelines to enable aircraft-based conflict detection and resolution (CD\&R) in the terminal maneuvering area (TMA) under current and emerging NextGen operating concepts providing an additional, protective safety layer for NextGen operations in the event that the tactical or strategic situation awareness is not sufficient or human errors or blunders occur. The CD\&R concepts use cockpit display designs to promote surface situation awareness and associated flight deck alerting concepts for safety assurance. The concepts employ continual own-ship and traffic data monitoring and algorithms to detect conflicts on the runway, at low altitudes near the airport, and during taxi and ramp operations for multiple classes of aircraft and surface vehicles. Alerts are generated as necessary and appropriate when traffic could affect runway safety or other TMA operational conditions that may require flight crew response.

Although substantial NASA research and testing has been conducted in the areas of surface operations situation awareness and runway incursion CD\&R [3, $4,5,6]$, much of this research has been conducted with a human-in-the-loop and has not included the effect of navigation accuracy and CD\&R system equipage levels on $C D \& R$ performance.

A fast-time batch simulation study was conducted to evaluate the performance of aircraftbased CD\&R algorithms in the TMA, with variations in surveillance accuracy. The algorithms were evaluated under various runway, taxiway and low altitude scenarios, multiple levels of CD\&R system equipage, and various levels of horizontal position accuracy. CD\&R performance was assessed through various metrics including the frequency of collisions and near-collisions and nuisance and missed alerts. This paper presents an overview of the CD\&R concept, description of the test method, and study results.

\section{System Description}

\section{Simulation Tool}

A simulation tool, known as Traffic Manager (TMX), was utilized for this study. TMX is a 
desktop simulation application designed for interaction studies of aircraft in present or future Air Traffic Management environments [7]. TMX can serve as a stand-alone traffic simulator, scenario generator, scenario editor, experiment control station, data recording tool, and rapid prototyping environment and can operate in real-time or fast-time mode. For this study, TMX was used in fast-time mode simulating various approach, departure, and taxi scenarios at the Chicago O'Hare International (KORD) airport. Although TMX is capable of simulating up to 2,000 aircraft simultaneously, only two aircraft per scenario were simulated. Each aircraft used a six-degree-of-freedom dynamics model.

Some modifications were made to TMX for this study. These included: 1) an updated database for the KORD airport; 2) creation of a Global Positioning System (GPS) sensor model for position accuracy; 3) creation of an interface to the CD\&R algorithm; 4) expansion of the pilot model to handle the required taxi, runway, and low altitude maneuvers; and various other minor modifications.

\section{Surveillance Data}

Traffic data are integral to the CD\&R concepts. For this study, it was assumed that Automatic Dependent Surveillance - Broadcast (ADS-B) would be used as the means for transmitting (ADS-B Out) and receiving (ADS-B In) aircraft surveillance data. ADS-B transmissions followed RTCA DO-242A specifications [8]; however, some simplifications were made to minimize computational overhead, such as not implementing models for latency effects and transmission line-of-sight and bandwidth blockage. For state-vector messages, a $1 \mathrm{~Hz}$ data transmission rate was specified. The position accuracy depended on the GPS measurement errors including a bias and an instantaneous jitter. A GaussMarkov process was used to model the time correlation between successive position measurement errors [9].

Navigation Accuracy Category for Position (NACp) describes the accuracy of positional information. NACp values range from 0 to 11 [8]. The horizontal Estimated Position Uncertainty (EPU) values for NACp categories of 8 and higher are listed in Table 1.
Table 1. NACp

\begin{tabular}{|c|c|}
\hline NACp & $95 \%$ Horizontal Accuracy Bound $($ EPU) \\
\hline 8 & EPU $<92.6 \mathrm{~m}(0.05 \mathrm{~nm}, 305.6 \mathrm{ft})$ \\
\hline 9 & EPU $<30 \mathrm{~m}(99 \mathrm{ft})$ \\
\hline 10 & EPU $<10 \mathrm{~m}(33 \mathrm{ft})$ \\
\hline 11 & EPU $<3 \mathrm{~m}(9.9 \mathrm{ft})$ \\
\hline
\end{tabular}

The Federal Aviation Administration (FAA) has issued an ADS-B Out Final Rule [10] which includes performance standards for ADS-B Out. The Rule states that EPU must be less than 0.05 nautical miles $(\mathrm{nm})$, which is equivalent to NACp 8.

Irrespective of this rule, Safety, Performance and Interoperability Requirements (SPR) for the Implementation of Enhanced Traffic Situational Awareness on the Airport Surface with Indications and Alerts (ATSA SURF IA) have proposed horizontal position accuracy requirements (RTCA SC-186 [11]). Through analysis, the SPR identified that to meet safety requirements, horizontal position accuracy on the airport surface needs to be at least 10 $\mathrm{m}$ within $95 \%$ containment bounds (NACp 10) to allow indications and alerts at virtually all airports in the National Airspace System. Validation of these requirements is on-going.

Based on the ADS-B Out Final Rule and SURF IA requirements, NACp values of $8,9,10$, and 11 were evaluated for this study. Truth data, with no accuracy errors, was also evaluated.

\section{Conflict Detection}

\section{CD\&R Algorithm}

The Airport Traffic Collision Avoidance Monitor (ATCAM) CD\&R algorithm was designed to identify potential traffic conflicts at low altitudes near the airport, on the runway, and during taxi and ramp operations for multiple classes of aircraft and surface vehicles.

ATCAM is comprised of three separate aircraftbased algorithms that rely on traffic state information obtained from ADS-B In:

1. The Runway Safety Monitor (RSM) is designed to detect and alert for runway conflicts. RSM monitors own-ship and traffic located in a three-dimensional virtual zone around the relevant runway using own-ship and traffic state data and separation and closure rate to determine whether an 
alert should be generated. RSM is described in detail in [12].

2. The Low Altitude Conflict Monitor (LACM) is designed to detect and alert for air-to-air conflicts near the airport at altitudes below $1000 \mathrm{ft}$ (i.e., to not conflict with the Traffic Alert and Collision Avoidance System (TCAS)). LACM computes closing speed, time to closest point of approach (CPA), time to co-altitude, and other data between own-ship and approaching aircraft to determine if criteria and thresholds are met for issuing alerts, similar to the TCAS approach.

3. The Taxi Conflict Monitor (TCM) is designed to detect and alert for ground taxi conflicts in the airport movement areas. The TCM design is similar to that of LACM and computes distances between own-ship and traffic, closing speeds, time to CPA and other parameters.

The three algorithms are independent but are integrated and share data to increase the probability of detection for all possible conflicts during airport TMA operations. RSM has been through extensive testing $[3,4,5,6]$. LACM and TCM are less mature but have been evaluated in simulation studies [13, 14]. Green, et al [15] provides a detailed description of ATCAM including initial alerting criteria.

\section{Alerts}

Alerts identify potential collision hazards which may require timely response by the flight crew to avoid a collision. A two-level alerting scheme is employed.

Caution alerts are generated for conditions that require immediate flight crew awareness and subsequent flight crew response. Generally, caution alerts are generated with sufficient time to evaluate the situation to be prepared to respond.

Warning alerts are generated for conditions that require immediate flight crew awareness and immediate flight crew response. Warning alerts may occur without preceding caution alerts.

The application of these alerts within the cockpit follows FAA Advisory Circular 25.1322-1.

\section{Test Method}

Data collection occurred for runway, taxi, and low altitude air-to-air conflict scenarios. Only two aircraft were included in each scenario to limit the interaction in this initial fast-time study. For ease of discussion, the aircraft will be referred to as Aircraft A and Aircraft B.

\section{CD\& $R$ Equipage}

Various levels of CD\&R system equipage were simulated for this study: a) both aircraft equipped; b) neither aircraft equipped; or, c) one or the other aircraft equipped.

When an aircraft was not equipped, it would follow its planned flight path to the end of the test run. When an aircraft was equipped, it would take action after a warning alert was generated by following an appropriate maneuver (e.g. go-around, abort, stop), depending on the operational phase. The maneuver was based on the relative location of the aircraft at the projected CPA.

\section{Test Scenarios}

Seven runway, three taxiway, and two low altitude air-to-air scenarios were developed. Variability was introduced into the scenarios by varying the location of the aircraft, speed of the aircraft, and/or time when the aircraft started to proceed along its predefined route. As a result, not every test run resulted in a conflict or collision. Due to space limitations, only two scenarios are reported in this document.

\section{Runway Scenario - Arrival with taxi crossing}

This scenario evaluated the situation where an aircraft was on approach when another aircraft taxied perpendicular across the runway.

The initial condition (IC) for Aircraft A was at $3.5 \mathrm{~nm}$ from the threshold at $1110 \mathrm{ft}$ above field level (AFL) at an indicated airspeed of $138 \mathrm{kt}$. Aircraft A flew at a constant altitude to intercept the glideslope at $3.3 \mathrm{~nm}$ from the threshold and then descended on a 3 degree glidepath for a straight-in approach to Runway 10. For the nominal flight plan, Aircraft A landed, decelerated at $2.5 \mathrm{~m} / \mathrm{s}$, taxied down the runway at $60 \mathrm{kt}$, and then slowed to exit the runway at Taxiway M7 at $3 \mathrm{kt}$. Aircraft B started at various locations around Runway 10 and taxied across starting from a complete stop and accelerating at 1 $\mathrm{m} / \mathrm{s} / \mathrm{s}$ to $15 \mathrm{kt}$. The actual taxiways for KORD were not used in this study. Instead, Aircraft B's initial position was placed at 14 different locations along 
the length of Runway $10(0,1000,1500,2000,2500$, $3000,3500,4000,4500,5000,6000,7500,9000$, and 10,000 feet from the runway threshold) simulating various taxiway entry points and at 18 locations away from the runway $(300,320,340,360,380,400,450$, $500,550,600,650,700,800,900,1,000,1,200$, 1,400 , and 1,600 feet from the runway centerline) see Figure 1. Aircraft B (red chevron, Figure 1) began to taxi when Aircraft A (blue chevron, Figure 1) was at various points in its approach and rollout (from $3.5 \mathrm{~nm}$ to the glidepath intercept point on the runway, at $0.5 \mathrm{~nm}$ intervals, crossing the runway threshold, glidepath aimpoint $(1000 \mathrm{ft})$, and $3000 \mathrm{ft}$, $4500 \mathrm{ft}, 1 \mathrm{~nm}, 8000 \mathrm{ft}$, and $9000 \mathrm{ft}$ past the threshold). If maneuvering were required based on a CD\&R warning alert being triggered in an appropriately equipped aircraft, Aircraft A would conduct a go-around if above $70 \mathrm{ft}$ AFL; otherwise, it would continue to land and stop on the runway. Aircraft B, if equipped, would stop if its nose had not reached the runway shoulder (greater than $100 \mathrm{ft}$ from the runway centerline) at the projected stopping point; otherwise, it would continue to taxi across the runway. When conducting an emergency stop by either aircraft, a $4 \mathrm{~m} / \mathrm{s} / \mathrm{s}$ deceleration rate was used.

\section{Taxi Scenario - Taxi intersection}

This scenario tested the situation where aircraft conflict at a perpendicular taxiway intersection. Aircraft A began taxi at $15 \mathrm{kt}$. Aircraft B taxied across the taxiway, starting from a complete stop and accelerating to $15 \mathrm{kt}$ at $1 \mathrm{~m} / \mathrm{s} / \mathrm{s}$. Aircraft B's initial position was placed at 4 different locations along Aircraft A's taxiway $(400,600,800$, and 1,000 feet ahead of Aircraft A) simulating various taxiway crossing points and at 5 locations away from the taxiway $(230,380,530,680$, and 830 feet from taxiway centerline) - see Figure 2. Aircraft B began to taxi when Aircraft $\mathrm{A}$ was at various locations along the taxiway $(0,150,300,450$, and $600 \mathrm{ft}$ from its starting position). If a warning alert was triggered on an equipped aircraft, the aircraft would stop at an accelerated rate $(4 \mathrm{~m} / \mathrm{s} / \mathrm{s})$ provided that it could be determined that the aircraft's nose location was greater than $100 \mathrm{ft}$ from the intersecting taxiway centerline at the projected stopping point; otherwise, it would continue to taxi across the taxiway.

\section{Pilot Reaction Delay}

A delay was incorporated to simulate the reaction time from when a pilot would receive a warning alert until action was taken to resolve the situation. The following delay times were used for this study: 5 seconds (sec) when aircraft was on approach, $3 \mathrm{sec}$ when aircraft was rolling out, and 2 sec during taxi. These delay times were selected based on reaction delays experienced during previous piloted simulation and flight testing.

\section{Test Matrix}

CD\&R algorithm performance was evaluated for the conflict scenarios described above using the ATCAM CD\&R algorithm for five levels of surveillance accuracy (NACp of $8,9,10,11$, and truth), and four levels of CD\&R system equipage (neither aircraft equipped, only Aircraft A equipped, only Aircraft B equipped, and both aircraft equipped). Thus, 20 cases were examined for each of the scenarios.

The number of replicates for each treatment combination varied according to level of surveillance accuracy. As shown in Table 2, more replicates were conducted for the lower NACp values in order to increase the level of precision for estimating the true location of the aircraft.

\section{Table 2. Number of Replicates}

\begin{tabular}{|c|c|}
\hline NACp & Number of replicates \\
\hline 8 & 7 \\
\hline 9 & 6 \\
\hline 10 & 4 \\
\hline 11 & 3 \\
\hline Truth & 1 \\
\hline
\end{tabular}

Some of the test conditions from Figures 1 and 2 , in which a conflict would obviously not occur, were omitted in order to reduce the size of the test matrix.

Data were collected for the test runs in random order by scenario. For data manageability of the scenarios that required a large number of test runs, data were collected randomly grouped by level of surveillance accuracy and CD\&R system equipage. 


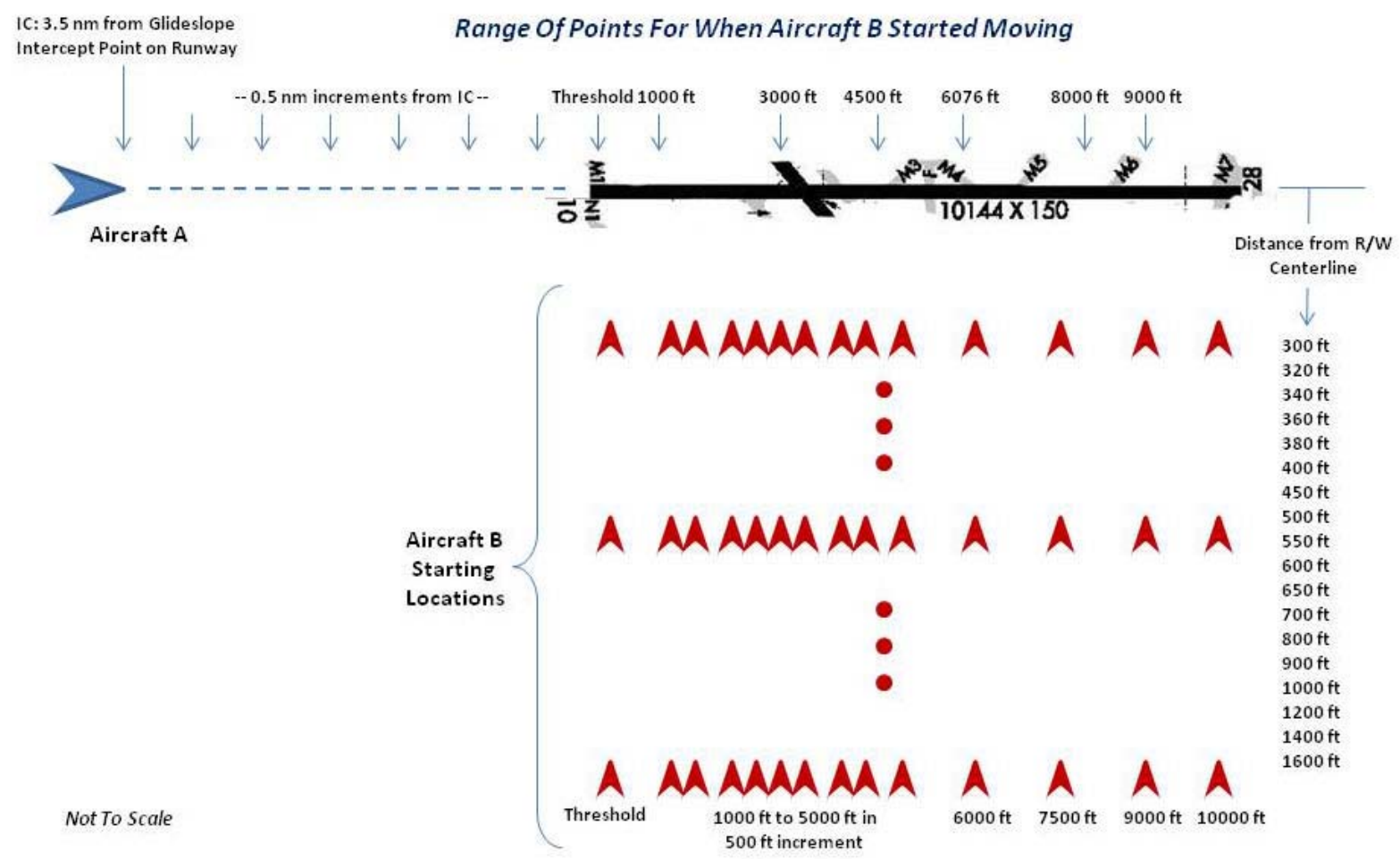

Figure 1. Runway Scenario - Arrival with Taxi Crossing Initial Conditions

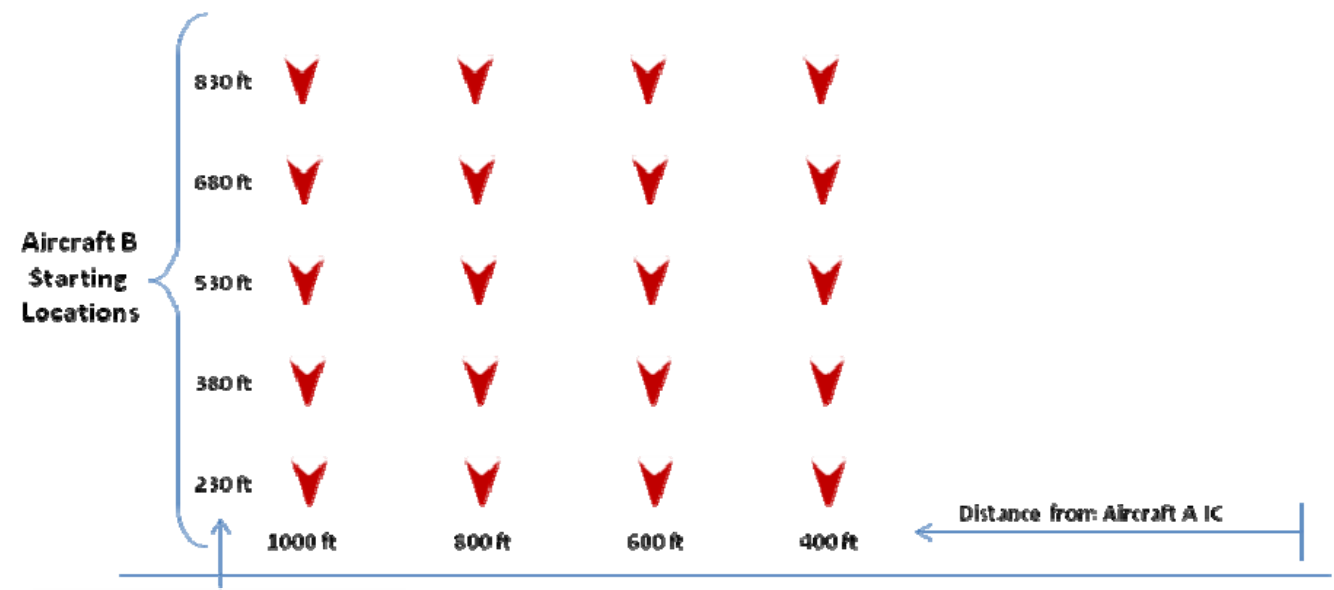

Distance from Taxiway Centerline

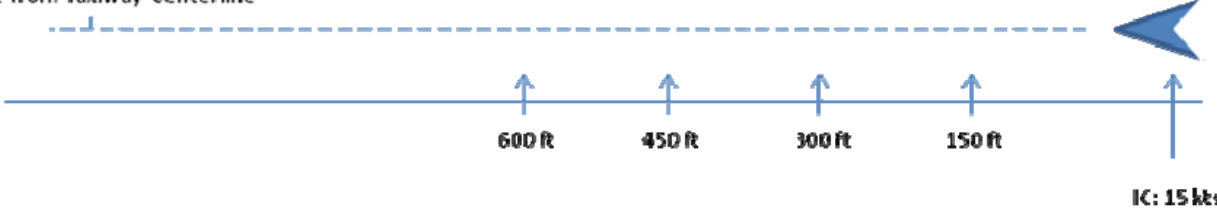

Aircraft A

Not To Scate

Range Of Points For When Aircraft B Started Moving

Figure 2. Taxi Scenario - Taxi Intersection Initial Conditions 


\section{Test Metrics}

Some of the metrics utilized for this study are defined in this section. All data is referenced from the aircraft center-of-gravity (CG), unless noted otherwise.

\section{Near-Collision / Collision}

For the runway scenario, a near-collision was counted if the CG's of the two aircraft were $<300 \mathrm{ft}$ apart laterally and vertical separation was $<200 \mathrm{ft}$. A collision was counted if the aircraft CG's were $<150$ $\mathrm{ft}$ apart laterally and vertical separation was $<100 \mathrm{ft}$.

For the taxiway scenario, a near-collision was counted if the aircraft CG's were $<185 \mathrm{ft}$ apart laterally. A collision was counted if the aircraft CG's were $<150 \mathrm{ft}$ apart laterally.

\section{Nuisance / Missed Alerts}

According to the SURF IA SPR [11], a nuisance alert is defined as any alert generated by a properly functioning CD\&R system that is inappropriate or unnecessary for the particular situation. Nuisance alerts could distract the flight crew unnecessarily, reduce confidence in the system, and negatively affect safety and operational effectiveness. Repeated nuisance alerts could decrease the use of CD\&R and reduce expeditious flight crew response to true alerts.

A missed alert is defined as a failure to provide an alert when it is necessary provided own-ship and traffic are adequately equipped [11]. Missed alerts represent a reduction in $C D \& R$ benefits.

The SURF IA SPR [11] definitions for nuisance and missed boundaries were applied. Horizontal position error was the only source of error modeled. Other sources of error, such as vertical position error, airport database error and flight technical error, were not included.

For the runway scenario, the following nuisance and missed boundary definitions applied for Aircraft A:

- When the aircraft was on approach, an approach corridor as defined in the SURF IA SPR for NACp 8 with a probability of missed alert of 0.01 was used since NACp 8 and higher was being evaluated. The corridor width was +/$321.5 \mathrm{ft}$ at the runway threshold and linearly increased to $+/-964.6 \mathrm{ft}$ at $3 \mathrm{~nm}$ away from the runway threshold. The nuisance boundary definition was when the true aircraft position was outside the approach corridor, but the detected position was within the approach corridor. The missed boundary definition was when the aircraft's true position was within the approach corridor, but the detected position was outside the approach corridor. Since the true position of the approach aircraft tracked the extended runway centerline, the aircraft could never enter the nuisance boundary.

- When the aircraft had crossed the runway threshold on landing or was traveling along a runway, the nuisance boundary definition was when the aircraft's true position was farther than one runway width $(150 \mathrm{ft})$ from the runway centerline, but the detected position was within one runway width of the centerline. The missed boundary definition was when the aircraft's true position was within one runway width of the runway centerline, but the detected position was greater than one runway width from the centerline.

For the runway scenario, the following nuisance and missed boundary definitions applied for Aircraft B:

- When the aircraft was taxiing across the runway, the nuisance boundary definition was when the true position of the aircraft's nose (when entering) or tail (when exiting) was at or behind the hold line, but any part of the detected aircraft (from nose to tail) was between the runway shoulder edges. The missed boundary definition was when the true position of any part of the aircraft was between the runway shoulder edges, but the detected nose position (when entering) or tail position (when exiting) was outside of the runway shoulder edges. A shoulder width of 7.5 $\mathrm{m}(25 \mathrm{ft})$, as defined in the SURF IA SPR for Aerodrome code 4, was used. A $150 \mathrm{ft}$ wide runway was assumed; therefore, the distance between shoulder edges was approximately 200 $\mathrm{ft}$. The hold line was located $225 \mathrm{ft}$ from the runway centerline.

For the taxi scenario, the following nuisance and missed boundary definitions applied for both aircraft:

- When the aircraft was traveling along the taxiway, the nuisance boundary definition was when the true position was farther than one 
taxiway width $(75 \mathrm{ft})$ from the taxiway centerline, but the detected position was within one taxiway width of the taxiway centerline. The missed boundary definition was when the true position was within one taxiway width of the taxiway centerline, but the detected position was greater than one taxiway width from the taxiway centerline. Since the true position of both aircraft during taxi tracked their respective taxiway centerlines, the aircraft could never enter the nuisance boundary.

- When the aircraft was crossing at a taxiway intersection, the nuisance boundary definition was when the true position of the nose (when entering) or tail (when exiting) was at or behind the taxiway holding position (assumed for this study to be $129.5 \mathrm{ft}$ from crossing taxiway centerline [17]), but any part of the detected aircraft (from nose to tail) was determined to be between the taxiway shoulder edges. A shoulder width of $25 \mathrm{ft}$ was used [18]. The missed boundary definition was when the true position of any part of the aircraft was determined to be between the taxiway shoulder edges, but the detected nose position (when entering) or tail position (when exiting) was outside of the taxiway shoulder edges.

Since only true vertical position was used, there was no opportunity for a nuisance or missed condition in the vertical direction.

An alert was considered to be a nuisance if the alert was generated when the aircraft was within a nuisance boundary, based on the definitions above.

A straight-forward corollary for a missed alert definition does not exist. If the aircraft was within the missed boundary, based on the definitions above, and an alert was not generated, that did not necessarily mean that an alert should have been generated. Even though one of the aircraft was in the missed boundary, the geometry of the aircrafts' trajectory may not be on a collision path. Therefore, if an alert was generated when using truth data but an alert was not generated at the same instance when using NACp data, then a missed alert was counted. This definition is algorithm dependent.

\section{Unwanted Alert}

This metric was developed as an effort to determine how far an alert zone should be from the hold line so unwanted (i.e. nuisance) alerts do not occur when an aircraft is preparing to cross the runway but is still behind the hold line. The edge of the alert zone was placed at the same location as the hold line (225 ft from the runway centerline) so an alert would be generated the moment the aircraft crossed the hold line. An alert was considered unwanted if the true aircraft position was behind the hold line but the detected position indicated the aircraft to be over the hold line, causing an alert. If an unwanted alert was triggered, the maximum distance the detected aircraft nose crossed over the hold line was recorded, thus approximating the distance the alert zone should be from the hold line.

\section{Results}

A summary of quantitative results is presented. All data is referenced from the aircraft CG, unless noted otherwise. For the aircraft used in this study, the nose position was $72.8 \mathrm{ft}$ from the $\mathrm{CG}$ and the tail position was $82 \mathrm{ft}$ from the CG.

For each scenario, the data analysis was limited to the area of interest, i.e., until the aircraft reached the CPA or until 10 seconds after a warning alert terminated, whichever was later. Also, both aircraft broadcasted the same level of positional accuracy for each test run.

Results are tabulated for NACp 8 to 11 and true position accuracy; however, the discussion of NACp 8 statistical results is limited due to poor performance based on positional error. The Kruskal-Wallis test was utilized to provide a non-parametric statistical hypothesis test to detect differences in NACp accuracy and CD\&R equipage using a significance level of $\alpha=0.05$ and $\mathrm{N}=$ number of test runs. When statistically significant differences were detected, post-hoc analysis was conducted using the nonparametric Mann-Whitney test.

\section{Runway Scenario - Arrival with taxi crossing}

For each of the 20 cases in this scenario, 2,367 combinations of the initiation delay and initial position for Aircraft B (taxiing aircraft) were evaluated, for a total of 198,828 test runs.

Algorithm performance - The data (Table 3) shows that warning alerts were generated on approximately $50 \%$ of the runs for either aircraft, 
almost independent of the NACp levels. Caution alerts were issued on approximately $50 \%$ of the runs for the taxiing aircraft (Aircraft B) but only 20\% of the time for the approach aircraft (Aircraft A). Caution alerts are issued on approach when the aircraft is $8,000 \mathrm{ft}$ to $6,000 \mathrm{ft}$ from the runway threshold. During a higher percentage of test runs, the alerting criteria was not met until the aircraft was less than $6,000 \mathrm{ft}$ from the runway threshold on approach or on the runway after landing, within the warning alert zone. The number of caution alerts for NACp 9 accuracy was statistically different from NACp 10, 11 and truth accuracies over all equipage levels. There was no statistical difference between accuracy levels when analyzing the number of warning alerts over all equipage levels.

Alert toggling occurred when multiple instances of caution and warning alerts were generated during a test run. Alert toggling is undesirable (i.e., it is a distraction to the flight crew and would cause mistrust in the technology).

As the position accuracy was reduced, alert toggling occurred more frequently, particularly for NACp 8 and 9 accuracies (see Table 3). The number of multiple caution and warning alerts for NACp 9 accuracy was statistically different from NACp 10 and 11 and truth accuracy over all equipage levels. This toggling included gaps between alerts in many instances. In addition to position accuracy, the toggling can also be a result of aircraft maneuvering. It should be noted that for Aircraft B, toggling occurred when accurate (true) position data was transmitted, which was not expected. It was determined that these multiple alerts were generated after Aircraft B had crossed Runway 10 and was entering a nearby runway. Alerts should not have been generated in this situation because there was not a traffic threat on the nearby runway.

Missed and nuisance alerts - The number of runs in which the aircraft entered the defined missed and nuisance boundaries increased as the position accuracy decreased, as shown in Table 4. Aircraft can cross into the missed and nuisance boundary multiple times throughout a test run, for varying lengths of time. The number of times (count) and amount of time (duration and percentage of run length) that the aircraft were within the boundaries was greater when using less accurate data (see Table 4). Although there was no statistical difference between accuracy levels for the number of times entering the missed and nuisance boundary across equipage levels, this trend was particularly evident when using NACp 8 accuracy.

For the approach aircraft (Aircraft A), the majority of occurrences of entering the missed boundary was after the aircraft had crossed the runway threshold for landing. The aircraft only entered the missed boundary while on approach before crossing the runway threshold during $2.8 \%$ of the test runs when using NACp 8 accuracy. Since Aircraft A tracked the extended centerline on approach and centerline after landing, the nuisance

Table 3. Alert Statistics by Aircraft for Arrival / Taxi Crossing Runway Scenario

\begin{tabular}{|c|c|c|c|c|c|}
\hline NACp & $\begin{array}{c}\text { Total \# } \\
\text { Runs }\end{array}$ & $\begin{array}{c}\text { Caution Alerts } \\
\text { (\% runs) }\end{array}$ & $\begin{array}{c}\text { Multiple Caution } \\
\text { Alerts } \\
\text { (\% runs) }\end{array}$ & $\begin{array}{c}\text { Warning Alerts } \\
\text { (\% runs) }\end{array}$ & $\begin{array}{c}\text { Multiple Warning } \\
\text { Alerts } \\
\text { (\% runs) }\end{array}$ \\
\hline \multicolumn{5}{|c|}{ Aircraft A } \\
\hline 8 & 66,276 & 29.84 & 3.86 & 50.51 & 15.74 \\
\hline 9 & 56,808 & 19.88 & 1.10 & 45.54 & 3.53 \\
\hline 10 & 37,872 & 16.22 & 0.01 & 44.34 & 0.14 \\
\hline 11 & 28,404 & 16.18 & 0.01 & 44.03 & 0.00 \\
\hline Truth & 9,468 & 16.14 & 0.00 & 43.94 & 17.64 \\
\hline \multicolumn{7}{|c|}{ Aircraft B } \\
\hline 8 & 66,276 & 57.75 & 19.44 & 44.36 & 5.56 \\
\hline 10 & 56,808 & 46.19 & 2.94 & 48.43 & 1.69 \\
\hline 11 & 28,404 & 43.34 & 1.81 & 47.55 & 1.35 \\
\hline Truth & 9,468 & 43.08 & 1.92 & 47.64 & 47.69 \\
\hline
\end{tabular}


Table 4. Missed and Nuisance Boundary Statistics by Aircraft for Arrival / Taxi Crossing Runway Scenario

\begin{tabular}{|c|c|c|c|c|c|c|c|c|}
\hline \multirow[b]{2}{*}{$\mathrm{NACp}$} & \multicolumn{4}{|c|}{ Entered Missed Boundary } & \multicolumn{4}{|c|}{ Entered Nuisance Boundary } \\
\hline & $\begin{array}{l}\text { \# runs, } \\
\text { \% runs }\end{array}$ & $\begin{array}{c}\text { Count } \\
\text { (weighted } \\
\text { mean, SD) }\end{array}$ & $\begin{array}{c}\text { Duration } \\
\text { (seconds) } \\
\text { (mean, SD) }\end{array}$ & \begin{tabular}{|c|}
$\%$ of \\
Run \\
Length
\end{tabular} & $\begin{array}{l}\text { \# runs, } \\
\% \text { runs }\end{array}$ & $\begin{array}{c}\text { Count } \\
\text { (weighted } \\
\text { mean, SD) }\end{array}$ & $\begin{array}{c}\text { Duration } \\
\text { (seconds) } \\
\text { (mean, SD) }\end{array}$ & $\begin{array}{c}\% \text { of } \\
\text { Run } \\
\text { Length }\end{array}$ \\
\hline \multicolumn{9}{|c|}{ Aircraft A } \\
\hline 8 & $54221,81.8$ & 6.6 & $11.4,11.9$ & \begin{tabular}{|l|}
7.8 \\
\end{tabular} & $377,0.6$ & 1.1 & 0.6 & 0.3 \\
\hline 9 & $662,1.2$ & 1.6 & $1.4, \quad 1.6$ & 0.8 & $335, \quad 0.6$ & $1.0,1.0$ & 0.6 & 0.2 \\
\hline 10 & $149,0.4$ & $1.0, \quad 1.0$ & 0.5 & 0.2 & $136, \quad 0.4$ & $1.0,0.0$ & 0.4 & 0.2 \\
\hline 11 & $63,0.2$ & 1.0 & 0.2 & 0.1 & $64, \quad 0.2$ & $1.0, \quad 0.0$ & 0.2 & 0.1 \\
\hline Truth & $0,0.0$ & 0.0 & 0.0 & 0.0 & $0, \quad 0.0$ & $0.0, \quad 0.0$ & 0.0 & 0.0 \\
\hline \multicolumn{9}{|c|}{ Aircraft B } \\
\hline 8 & $47923,72.3$ & 2.0 & $4.1,2.9$ & 3.6 & $19801,29.9$ & 3.6 & 6.5 & 5.6 \\
\hline 9 & $34867,61.4$ & 1.3, & $1.5,1.2$ & 1.3 & $87, \quad 0.2$ & $\begin{array}{ll}1.7, & 0.9\end{array}$ & 1.3, & 1.1 \\
\hline 10 & $20852,55.1$ & 1.1 & $0.6, \quad 0.3$ & 0.5 & $0, \quad 0.0$ & 0.0 & 0.0 & 0.0 \\
\hline 11 & $10090,35.5$ & 1.1 & $0.3,0.1$ & 0.2 & 0.0 & 0.0 & 0.0 & 0.0 \\
\hline Truth & $0, \quad 0.0$ & $0.0, \quad 0.0$ & $0, \quad 0.0$ & 0.0 & $\begin{array}{ll}0, & 0.0\end{array}$ & $0.0, \quad 0.0$ & $0, \quad 0.0$ & 0.0 \\
\hline
\end{tabular}

boundary was entered as the aircraft was exiting the runway.

The taxiing aircraft (Aircraft B) entered the missed boundary at least once for a high percentage of the test runs for accuracy levels of NACp 8 to 11 . This was due to the criteria for entering the missed boundary. The aircraft was counted as entering the missed boundary when the true position of any part of the aircraft was determined to be between the runway shoulder edges, but the detected nose position (when entering) or tail position (when exiting) was outside of the runway shoulder edges. As such, there was no buffer between when the aircraft was inside or outside the missed boundary so a measurable difference between the true and detected position could cause a missed boundary to be counted.

The number of test runs that contained missed and nuisance alerts was relatively low, overall, as shown in Table 5. The missed alert definition, as noted earlier, is algorithm dependent. Missed alerts for both aircraft were highest when using NACp 8 accuracy. There was no statistical difference between accuracy levels when analyzing missed caution alerts and missed warning alerts over all equipage levels. Aircraft A did, however, experience two missed alerts under the truth accuracy condition.
This unexpected event occurred because even though the aircraft were broadcasting true position data, the ADS-B transmission model was still being used. The transmission model resulted in a slight delay between the aircraft's position at the time of transmitting the ADS-B message and the position at the time of reception of the ADS-B message by Aircraft B. This delay was present in all scenarios, but this position difference was negligible compared to the NACp position uncertainty error. In these two scenarios, Aircraft B did not detect a conflict with Aircraft A based on the broadcast position, but if instantaneous position information were used for Aircraft A, a conflict would have been detected. The small error introduced by the movement of Aircraft A between transmission and reception of the ADS-B message resulted in just enough difference in relation to Aircraft B's position to result in the missed alerts. For Aircraft A, all of the missed alerts, for all accuracy levels, occurred after the aircraft had crossed the runway threshold for landing. An alert was considered a nuisance if it was generated at the same time the aircraft was determined to be within the nuisance boundary. Therefore, nuisance alerts for Aircraft A only occurred as the aircraft was exiting the runway (see explanation above). 
Table 5. Missed and Nuisance Alert Statistics by Aircraft for Arrival / Taxi Crossing Runway Scenario

\begin{tabular}{|c|c|c|c|c|c|}
\hline NACp & $\begin{array}{c}\text { Total \# } \\
\text { Runs }\end{array}$ & $\begin{array}{c}\text { Missed } \\
\text { Caution Alerts } \\
\text { (\% runs) }\end{array}$ & $\begin{array}{c}\text { Missed } \\
\text { Warning Alerts } \\
\text { (\% runs) }\end{array}$ & $\begin{array}{c}\text { Nuisance } \\
\text { Caution Alerts } \\
\text { (\% runs) }\end{array}$ & $\begin{array}{c}\text { Nuisance } \\
\text { Warning Alerts } \\
\text { (\% runs) }\end{array}$ \\
\hline \multicolumn{5}{|c|}{ Aircraft A } \\
\hline 8 & 66,276 & 0.10 & 5.97 & 0.01 & 0.03 \\
\hline 9 & 56,808 & 0.02 & 1.64 & 0.00 & 0.01 \\
\hline 10 & 37,872 & 0.02 & 0.04 & 0.00 & 0.00 \\
\hline 11 & 28,404 & 0.01 & 0.01 & 0.00 & 0.00 \\
\hline Truth & 9,468 & 0.00 & 0.00 & 0.00 & 2.03 \\
\hline 8 & 66,276 & 1.90 & Aircraft B & $0.00 *$ \\
\hline 9 & 56,808 & 0.68 & 4.34 & 0.74 & 0.00 \\
\hline 10 & 37,872 & 0.22 & 0.26 & 0.00 & 0.00 \\
\hline 11 & 28,404 & 0.10 & 0.07 & 0.00 & 0.00 \\
\hline Truth & 9,468 & 0.02 & 0.00 & 0.00 & \\
\hline
\end{tabular}

* Only one occurrence

Unnecessary maneuvering - Previous research has shown that pilots instinctively react upon receiving airport traffic warning alerts in the flight deck [14] without necessarily confirming with secondary or additional information first. It is critical that alerting only occurs when needed; otherwise, these unnecessary maneuvers can cause delays, equipment wear, and other costs to airlines.

To evaluate this situation, maneuvering was considered unnecessary if made based on a warning alert issued when the aircraft were broadcasting NACp accuracy, but for the same test conditions, a warning alert was not issued when broadcasting true position data. This measure quantifies untimely nuisance alerts using an algorithm-dependent methodology.

The percentage of test runs in which the aircraft maneuvered unnecessarily when using NACp data accuracy is shown in Table 6. Thus, as the accuracy decreased, the frequency of occurrences of unnecessary maneuvers increased. With a NACp value of 8 , approximately $10 \%$ or 1 in 10 of the warning alerts were unnecessary. With a NACp value of 11, approximately 1 in 1000 warning alerts were unnecessary for Aircraft A and 2 in 1000 was unnecessary for Aircraft B. There was no statistical difference between accuracy levels when analyzing unnecessary maneuvers over all equipage levels.
Table 6. Unnecessary Maneuvers by Aircraft during Arrival / Taxi Crossing Runway Scenario

\begin{tabular}{|c|c|c|}
\hline NACp & $\begin{array}{c}\text { Aircraft A } \\
\text { (\% runs) }\end{array}$ & $\begin{array}{c}\text { Aircraft B } \\
\text { (\% runs) }\end{array}$ \\
\hline 8 & 10.8 & 9.5 \\
\hline 9 & 2.8 & 3.7 \\
\hline 10 & 0.3 & 0.4 \\
\hline 11 & 0.1 & 0.2 \\
\hline
\end{tabular}

Collision avoidance - By the design of the scenarios, approximately $20 \%$ of the runs resulted in a near collision (NC) and approximately $10 \%$ resulted in collisions (C) in the absence of CD\&R, as shown in Table 7 . Collision avoidance was significantly affected by the CD\&R system equipage level. The most collisions were avoided when both aircraft were equipped with CD\&R. In some instances, CD\&R was more effective depending on which aircraft was equipped. For this scenario, more collisions were avoided when the crossing traffic (Aircraft B) was equipped. However, when only the approach aircraft (Aircraft A) was equipped, collision avoidance was less effective, but better that when neither aircraft were equipped. Many collisions were unavoidable for the approach aircraft. The most frequent causes were the warning alert occurred during high speed rollout or when the aircraft was too close to the ground to go around; other times the 
Table 7. Percentage of Near-Collisions (NC) and Collisions (C) for Equipage Combinations for Arrival / Taxi Crossing Runway Scenario

\begin{tabular}{|l|c|c|c|c|c|c|c|c|c|}
\hline & & \multicolumn{9}{|c|}{ CD\&R Equipage } \\
\cline { 3 - 10 } \#ACp & E Runs per & \multicolumn{2}{|c|}{ Neither } & \multicolumn{2}{c|}{ Aircraft A } & \multicolumn{2}{c|}{ Aircraft B } & \multicolumn{2}{c|}{ Both } \\
\cline { 3 - 10 } NAuipage & NC & C & NC & C & NC & C & NC & C \\
\hline 8 & 16,569 & 19.3 & 9.3 & 15.2 & 6.4 & 15.8 & 3.1 & 10.5 & 2.6 \\
\hline 9 & 14,202 & 19.3 & 9.3 & 16.3 & 7.0 & 16.6 & 0.8 & 9.4 & 0.5 \\
\hline 10 & 9,468 & 19.3 & 9.3 & 16.4 & 7.1 & 17.2 & 0.6 & 10.2 & 0.2 \\
\hline 11 & 7,101 & 19.3 & 9.3 & 16.4 & 7.1 & 17.5 & 0.3 & 10.6 & 0.1 \\
\hline Truth & 2,367 & 19.3 & 9.3 & 16.5 & 7.2 & 17.5 & 0.3 & 10.8 & 0.0 \\
\hline
\end{tabular}

Table 8. Unwanted Alert Data for Arrival / Taxi Crossing Runway Scenario

\begin{tabular}{|c|c|c|c|c|c|}
\hline \multirow{2}{*}{ NACp } & \multirow{2}{*}{$\begin{array}{c}\text { Total \# } \\
\text { Runs }\end{array}$} & \multicolumn{2}{|c|}{ Unwanted Caution } & \multicolumn{2}{c|}{ Unwanted Warning } \\
\cline { 3 - 7 } & & $\begin{array}{c}\text { Max dist over hold line } \\
\text { (ft) (mean, st dev) }\end{array}$ & \% Runs & $\begin{array}{c}\text { Max dist over hold line } \\
(\mathrm{ft})(\mathrm{mean}, \mathrm{st} \text { dev })\end{array}$ \\
\hline 8 & 66,276 & 8.9 & $126.4,75.3$ & 17.4 & $147.5,80.7$ \\
\hline 9 & 56,808 & 2.1 & $50.5,26.4$ & 6.7 & $55.4,26.0$ \\
\hline 10 & 37,872 & 0.1 & $16.6,11.2$ & 0.4 & $24.7, \quad 9.5$ \\
\hline 11 & 28,404 & 0.0 & $0.0, \quad 0.0$ & 0.0 & $0.0,0.0$ \\
\hline Truth & 9,468 & 0.0 & $0.0, \quad 0.0$ & 0.0 & $0.0,0.0$ \\
\hline
\end{tabular}

collision occurred after the maneuver was initiated but before the aircraft started climbing or during climb-out or during taxi on the runway. In some instances, the approach aircraft landed, exited the runway, and stopped but then the taxiing aircraft collided with it. Position accuracy had little effect on collision avoidance, with no statistical difference in collision avoidance between position accuracy levels. However, there were more collisions when using NACp 8 accuracy when either Aircraft B or both aircraft were equipped.

Unwanted alert - As defined above, an alert was considered unwanted if the true aircraft position (nose) for Aircraft B was behind the hold line but the detected position indicated that the aircraft was over the hold line, causing an alert. The frequency of unwanted alerts increased as the position accuracy decreased, particularly when using NACp 8 accuracy, as shown in Table 8. Also, a larger buffer between the hold line and alerting zone is required as the position accuracy decreases in order to reduce the number of unwanted alerts (see Table 8). For the arrival with taxi crossing scenario, 99 percent of unwanted alerts could have been avoided by placing the hold line $390 \mathrm{ft}$ from the hold line for NACp 8 accuracy, $135 \mathrm{ft}$ from the hold line for NACp 9 accuracy, and $55 \mathrm{ft}$ from the hold line for NACp 10 accuracy. The maximum standard for separation between the hold line and runway centerline is $280 \mathrm{ft}$ [17] to accommodate the largest aircraft. A $390 \mathrm{ft}$ alerting zone buffer is not practical for even the largest airports.

\section{Taxi Scenario - Taxi intersection}

For each of the 20 cases in this scenario, 76 combinations of the initiation delay and initial location for Aircraft B were evaluated, for a total of 6,384 test runs.

Algorithm performance - The data shows (Table 9) that caution alerts were generated for $55 \%$ to $76 \%$ of the test runs for both aircraft and warning alerts were generated for $50 \%$ to $66 \%$ of the runs. The number of caution and warning alerts for NACp 9 accuracy was statistically different from NACp 10, 11 and truth accuracies over all equipage levels.

As the position accuracy was reduced, alert toggling occurred more frequently, especially when using NACp 8 and 9 accuracies (see Table 9). The number of multiple caution and warning alerts for 
Table 9. Alert Statistics by Aircraft for Taxi Intersection Scenario

\begin{tabular}{|c|c|c|c|c|c|}
\hline NACp & $\begin{array}{c}\text { Total \# } \\
\text { Runs }\end{array}$ & $\begin{array}{c}\text { Caution Alerts } \\
\text { (\% runs) }\end{array}$ & $\begin{array}{c}\text { Multiple Caution } \\
\text { Alerts } \\
\text { (\% runs) }\end{array}$ & $\begin{array}{c}\text { Warning Alerts } \\
\text { (\% runs) }\end{array}$ & $\begin{array}{c}\text { Multiple Warning } \\
\text { Alerts } \\
\text { (\% runs) }\end{array}$ \\
\hline \multicolumn{5}{|c|}{ Aircraft A } \\
\hline 8 & 2,128 & 76.6 & 36.5 & 66.8 & 21.1 \\
\hline 9 & 1,824 & 70.5 & 16.3 & 61.2 & 3.9 \\
\hline 10 & 1,216 & 58.1 & 3.2 & 53.7 & 0.1 \\
\hline 11 & 912 & 56.4 & 0.1 & 51.4 & 0.0 \\
\hline Truth & 304 & 55.6 & 0.0 & 50.0 & 15.5 \\
\hline \multicolumn{7}{|c|}{ Aircraft B } \\
\hline 8 & 2,128 & 57.3 & 14.5 & 63.8 & 0.0 \\
\hline 9 & 1,824 & 56.5 & 1.8 & 57.7 & 0.0 \\
\hline 10 & 1,216 & 55.3 & 0.0 & 53.5 & 0.0 \\
\hline 11 & 912 & 56.3 & 0.0 & 51.3 & 50.0 \\
\hline Truth & 304 & 58.6 & 0.0 &
\end{tabular}

Table 10. Missed and Nuisance Boundary Statistics by Aircraft for Taxi Intersection Scenario

\begin{tabular}{|c|c|c|c|c|c|c|c|c|}
\hline \multirow[b]{2}{*}{ NACp } & \multicolumn{4}{|c|}{ Entered Missed Boundary } & \multicolumn{4}{|c|}{ Entered Nuisance Boundary } \\
\hline & $\begin{array}{l}\text { \# runs, } \\
\% \text { runs }\end{array}$ & $\begin{array}{c}\text { Count } \\
\text { (weighted } \\
\text { mean, SD) }\end{array}$ & $\begin{array}{c}\text { Duration } \\
\text { (seconds) } \\
\text { (mean, SD) }\end{array}$ & $\begin{array}{l}\% \text { of } \\
\text { Run } \\
\text { Length }\end{array}$ & $\begin{array}{l}\text { \# runs, } \\
\% \text { runs }\end{array}$ & $\begin{array}{c}\text { Count } \\
\text { (weighted } \\
\text { mean, SD) }\end{array}$ & $\begin{array}{c}\text { Duration } \\
\text { (seconds) } \\
\text { (mean, SD) }\end{array}$ & $\begin{array}{l}\% \text { of } \\
\text { Run } \\
\text { Length }\end{array}$ \\
\hline \multicolumn{9}{|c|}{ Aircraft A } \\
\hline 8 & 2128,100 & $8.1,7.8$ & $18.6,11.0$ & 54.2 & $703,33.0$ & $1.8, \quad 1.1$ & $3.4,5.2$ & 9.6 \\
\hline 9 & $1339,73.4$ & $2.2, \quad 1.1$ & $3.1,4.4$ & 9.2 & $62, \quad 3.4$ & $1.1, \quad 1.0$ & $1.0,1.8$ & 2.8 \\
\hline 10 & $666,54.8$ & $1.1, \quad 0.9$ & $0.5, \quad 0.3$ & 1.5 & $0, \quad 0.0$ & $0.0, \quad 0.0$ & $0,0.0$ & 0.0 \\
\hline 11 & $327,35.9$ & $1.0, \quad 1.0$ & $0.3, \quad 0.1$ & 0.8 & $0, \quad 0.0$ & $0.0, \quad 0.0$ & $0,0.0$ & 0.0 \\
\hline Truth & $0, \quad 0.0$ & 0.0 & 0.0 & 0.0 & $0, \quad 0.0$ & 0.0 & $0,0.0$ & 0.0 \\
\hline \multicolumn{9}{|c|}{ Aircraft B } \\
\hline 8 & 2128,100 & $8.0, \quad 7.5$ & $5.3, \quad 6.1$ & 15.3 & $674,31.7$ & $1.9, \quad 1.2$ & $3.8,5.2$ & 11.1 \\
\hline 9 & $1124,61.6$ & $2.2, \quad 1.2$ & $2.7, \quad 3.9$ & 8.2 & $53, \quad 2.9$ & $1.2, \quad 0.9$ & $0.9,0.9$ & 2.6 \\
\hline 10 & $430,35.4$ & $1.0, \quad 1.0$ & $0.5, \quad 0.3$ & 1.7 & $0, \quad 0.0$ & $0.0, \quad 0.0$ & $0,0.0$ & 0.0 \\
\hline 11 & $197,21.6$ & 1.0 & $0.3, \quad 0.1$ & 0.9 & 0.0 & 0.0 & $0,0.0$ & 0.0 \\
\hline Truth & $\begin{array}{ll}0, & 0.0 \\
\end{array}$ & $0.0, \quad 0.0$ & $0.0, \quad 0.0$ & 0.0 & $\begin{array}{ll}0, & 0.0 \\
\end{array}$ & $0.0, \quad 0.0$ & $0,0.0$ & 0.0 \\
\hline
\end{tabular}

NACp 9 accuracy was statistically different from NACp 10 and 11 and truth accuracies over all equipage levels. For Aircraft $\mathrm{A}$, the number of multiple caution alerts for NACp 9 accuracy was statistically different from NACp 10 accuracy; furthermore, NACp 10 accuracy was statistically different from NACP 11 and truth accuracies over all equipage levels.

Missed and nuisance alerts - For a large percentage of test runs, the aircraft entered the defined missed boundary for all surveillance accuracies, except truth, as shown in Table 10. The aircraft entered the missed boundary for all test runs when using NACp 8 accuracy and over 60 percent of runs when using NACp 9 accuracy. The number of times (count) and amount of time (duration and percentage of run length) that the aircraft were within the missed boundary was greater when using less accurate data (see Table 10). This was particularly true when using NACp 8 accuracy. There was a 
Table 11. Missed and Nuisance Alert Statistics by Aircraft for Taxi Intersection Scenario

\begin{tabular}{|c|c|c|c|c|c|}
\hline NACp & $\begin{array}{c}\text { Total \# } \\
\text { Runs }\end{array}$ & $\begin{array}{c}\text { Missed } \\
\text { Caution Alerts } \\
\text { (\% runs) }\end{array}$ & $\begin{array}{c}\text { Missed } \\
\text { Warning Alerts } \\
\text { (\% runs) }\end{array}$ & $\begin{array}{c}\text { Nuisance } \\
\text { Caution Alerts } \\
\text { (\% runs) }\end{array}$ & $\begin{array}{c}\text { Nuisance } \\
\text { Warning Alerts } \\
\text { (\% runs) }\end{array}$ \\
\hline \multicolumn{7}{|c|}{ Aircraft A } \\
\hline 8 & 2,128 & 5.7 & 6.5 & 0.0 & 0.0 \\
\hline 9 & 1,824 & 3.0 & 1.9 & 0.0 & 0.0 \\
\hline 10 & 1,216 & 2.5 & 0.6 & 0.0 & 0.0 \\
\hline 11 & 912 & 0.8 & 0.0 & 0.0 & 0.0 \\
\hline Truth & 304 & 0.0 & 0.0 & 0.0 & 0.0 \\
\hline \multicolumn{7}{|c|}{ Aircraft B } \\
\hline 8 & 2,128 & 5.7 & 6.5 & 0.0 & 0.0 \\
\hline 9 & 1,824 & 3.0 & 1.9 & 0.0 & 0.0 \\
\hline 10 & 1,216 & 2.5 & 0.6 & 0.0 & 0.0 \\
\hline 11 & 912 & 0.8 & 0.0 & 0.0 & 0.0 \\
\hline Truth & 304 & 0.0 & 0.0 & \\
\hline
\end{tabular}

statistically significant difference between NACp accuracy when analyzing the number of times entering the missed boundary and nuisance boundary over all equipage levels. The post-hoc analysis revealed NACp 9 accuracy was statistically different from NACp 10 and 11 and truth accuracies. The aircraft entered the missed boundary when both crossing the intersecting taxiway and when traveling along the taxiway. As with the previous scenario, the high rate of crossing the missed boundary was due to the missed boundary definition. There was no buffer between when the aircraft was inside or outside the missed boundary; therefore, a very small difference between the true and detected position caused a missed boundary to be counted. The aircraft entered the nuisance boundary only for NACp 8 and 9 accuracies. Since the aircraft's true taxi path was along the taxiway centerline, it was only possible to enter the nuisance boundary when crossing the intersecting taxiway.

The number of test runs that contained missed alerts increased as the position accuracy decreased, as shown in Table 11. Missed alerts for both aircraft were highest when using NACp 8 accuracy (less than 7 percent of test runs). There was no statistical difference between accuracy levels when analyzing missed caution alerts and missed warning alerts over all equipage levels. The rate of missed alerts was the same for both aircraft since the aircraft conducted identical operations, traveling along and crossing a taxiway. It was only possible for a nuisance alert to be generated when taxiing across a taxiway; however, there were not any nuisance alerts for either aircraft for this test scenario.

Unnecessary maneuvering - For the test runs in which both aircraft were broadcasting NACp accuracies and required to maneuver based on a warning alert, the percentage of test runs in which the aircraft maneuvered unnecessarily is shown in Table 12. Thus, as the data accuracy decreased, the frequency of occurrences of unnecessary maneuvering increased. With a NACp value of 8 , over one third of the warning alerts were unnecessary. With a NACp value of 11 , less than 3 in 100 warning alerts were unnecessary for Aircraft $A$ and less than 2 in 100 were unnecessary for Aircraft B. There was no statistical difference between accuracy levels when analyzing unnecessary maneuvers over all equipage levels.

Table 12. Unnecessary Maneuvers during Taxi Intersection Scenario

\begin{tabular}{|c|c|c|}
\hline NACp & $\begin{array}{c}\text { Aircraft A } \\
\text { (\% runs) }\end{array}$ & $\begin{array}{c}\text { Aircraft B } \\
\text { (\% runs) }\end{array}$ \\
\hline 8 & 38.0 & 36.8 \\
\hline 9 & 19.5 & 14.9 \\
\hline 10 & 4.6 & 3.9 \\
\hline 11 & 2.6 & 1.8 \\
\hline
\end{tabular}


Table 13. Percentage of Near-Collisions (NC) and Collisions (C) for Equipage Combinations for Taxi Intersection Scenario

\begin{tabular}{|l|c|c|c|c|c|c|c|c|c|}
\hline \multirow{2}{*}{ \# Runs per } & \multicolumn{9}{|c|}{ CD\&R Equipage } \\
\cline { 3 - 10 } NACp & Equipage & \multicolumn{2}{|c|}{ Neither } & \multicolumn{2}{c|}{ Aircraft A } & \multicolumn{2}{c|}{ Aircraft B } & \multicolumn{2}{c|}{ Both } \\
\hline 8 & 532 & 50.0 & 43.4 & 29.3 & 20.1 & 31.6 & 23.7 & 23.1 & 16.9 \\
\hline 9 & 456 & 50.0 & 43.4 & 28.5 & 19.5 & 26.3 & 16.9 & 10.7 & 4.4 \\
\hline 10 & 304 & 50.0 & 43.4 & 29.6 & 18.8 & 21.7 & 13.8 & 5.3 & 1.0 \\
\hline 11 & 228 & 50.0 & 43.4 & 29.4 & 18.4 & 20.2 & 14.0 & 2.2 & 0.0 \\
\hline Truth & 76 & 50.0 & 43.4 & 30.3 & 18.4 & 19.7 & 13.2 & 2.6 & 0.0 \\
\hline
\end{tabular}

Collision avoidance - For this scenario, CD\&R equipage was effective for collision avoidance, as shown in Table 13. Equipage for either aircraft dropped the near collision and collision rates in half, and with both aircraft equipped, the near collision rate was reduced by an order of magnitude and down to a $1 \%$ collision rate with NACp 10 accuracy. The data shows that more collisions were avoided more effectively when both aircraft were equipped with CD\&R; however, collisions still occurred, except when using very accurate data. Since both aircraft conducted identical operations, it was expected that collision avoidance would be similar for both aircraft. However, collision avoidance was slightly more effective when Aircraft B was equipped than when Aircraft A was equipped, except for NACp 8 accuracy. Position accuracy did not have much effect on collision avoidance, except when both aircraft were equipped. Over all levels of NACp accuracy, the differences in the number of collisions showed no statistically significant difference, when excluding NACp 8 accuracy; however, there were significant differences between the interaction of NACp accuracy and equipage levels. When only one aircraft was equipped (either A or B), there was no statistical difference in the number of collisions. When neither aircraft were equipped, there was a significant difference as the number of collisions was higher than when only one aircraft was equipped. When both aircraft were equipped, there was a significant difference in the number of collisions as they were lower than when only one aircraft was equipped.

Preliminary investigation was performed on the test runs that resulted in collision for the truth data condition. It was determined that for these test runs, warning alerts were generated; however, by the time the pilot reaction delay ( 2 seconds) was over, the aircraft was projected to stop closer than $100 \mathrm{ft}$ from the intersecting taxiway centerline. As a result, the aircraft continued taxi and a collision occurred.

\section{Summary}

A CD\&R concept for the airport TMA was evaluated in a fast-time batch simulation study. The purpose of the study was to evaluate the performance of an aircraft-based CD\&R algorithm during various runway, taxiway, and low altitude scenarios, multiple levels of CD\&R system equipage, and various levels horizontal position accuracy.

It is critical that alerting does not cause the flight crew to make unnecessary maneuvers since these actions can cause delays, equipment wear, and other costs to airlines. Results showed that for the scenarios reported herein, maneuvers were frequently made unnecessarily and were affected by position accuracy, with more unnecessary maneuvers occurring as position accuracy decreased.

Alert toggling occurs when multiple instances of caution and warning alerts are generated as a result of position accuracy or aircraft maneuvering. Alert toggling can be a distraction to the flight crew and could cause mistrust in the technology. In general, alert toggling occurred more frequently as the position accuracy was reduced, especially for NACp 8 and NACp 9 accuracy levels.

Missed alerts represent a reduction in CD\&R benefits and will result in operations such as they currently exist, where CD\&R alerts are not provided. 
The occurrence of missed alerts was much higher with NACp 8 position accuracy, in general.

Nuisance alerts could distract the flight crew unnecessarily, reduce confidence in the system, and can negatively affect safety and operational effectiveness. For the operations in which nuisance alerts were possible, the occurrence of nuisance alerts was relatively low for the scenarios reported on herein.

In general, collision avoidance was affected by the CD\&R equipage level. The most collisions were avoided if both aircraft were equipped with $C D \& R$. When only one aircraft was equipped with CD\&R, collision avoidance was more effective depending on which aircraft was equipped for the arrival with crossing taxi traffic scenario. CD\&R was not extremely effective for avoidance of near collisions for the arrival with taxi crossing scenario.

In order to reduce the number of unwanted alerts when taxiing across a runway, a buffer is needed between the hold line and the alerting zone so alerts are not generated when an aircraft is behind the hold line. Testing indicated that as the position accuracy decreases, a larger buffer is required. With NACp 8 data accuracy, such a large buffer would be required that it is not practical.

Continued analysis of these data is being conducted to identify horizontal positional accuracy requirements for effective terminal maneuvering area CD\&R and efficient algorithm designs.

\section{References}

[1] Joint Planning \& Development Office, 2010, Executive Summary, Integrated Work Plan for the Next Generation Air Transportation System, Version FY13.

[2] Joint Planning \& Development Office, 2010, Concept of Operations for the Next Generation Air Transportation System, Version 3.2.

[3] Jones, D. R., C. C. Quach, and S. D. Young, 2001, Runway Incursion Prevention System Demonstration and Testing at the Dallas/Fort Worth International Airport, Proceedings of the $20^{\text {th }}$ Digital Avionics Systems Conference.

[4] Jones, D. R., 2002, Runway Incursion Prevention System Simulation Evaluation, Proceedings of the
AIAA/IEEE $21^{\text {st }}$ Digital Avionics Systems Conference.

[5] Jones, D. R., 2005, Runway Incursion Prevention System Testing at the Wallops Flight Facility, Proceedings of the SPIE Defense \& Security Symposium.

[6] Jones, D. R. and L. J. Prinzel, 2006, Runway Incursion Prevention for General Aviation Operations, Proceedings of the AIAA/IEEE $25^{\text {th }}$ Digital Avionics Systems Conference.

[7] Bussink, F. J. L., J. Hoekstra, W. Heesbeen, 2005, Traffic Manager: A Flexible Desktop Simulation Tool Enabling Future ATM Research, Proceedings of the AIAA/IEEE $24^{\text {th }}$ Digital Avionics Systems Conference

[8] RTCA, Inc., 2002, Minimum Aviation System Performance Standards for Automatic Dependent Surveillance Broadcast (ADS-B), DO-242A.

[9] Mohleji, S. C., G. Wang, 2010, Modeling ADS-B Position and Velocity Errors for Airborne Merging and Spacing in Interval Management Application, MITRE release \# 10-3026.

[10] Department of Transportation Federal Aviation Administration, 2010, Automatic Dependent Surveillance - Broadcast (ADS-B) Out Performance Requirements to Support Air Traffic Control (ATC) Service; Final Rule, Docket No. FAA-2007-29305; Amdt. No. 91-314.

[11] RTCA, Inc., 2010, Safety, Performance and Interoperability Requirements Document for Enhanced Traffic Situational Awareness on the Airport Surface with Indications and Alerts (SURF IA), D0-323.

[12] Green, D. F., February 2006, Runway Safety Monitor Algorithm for Single and Crossing Runway Incursion Detection and Alerting, NASA CR-2006214275.

[13] Jones, D. R., L. J. Prinzel, S. D. Otero, and G. D. Barker, 2009, Collision Avoidance for Airport Traffic Concept Evaluation, Proceedings of the AIAA/IEEE $28^{\text {th }}$ Digital Avionics Systems Conference.

[14] Jones, D. R., L. J. Prinzel, K. J. Shelton, R. E. Bailey, S. D. Otero, and G. D. Barker, 2010, Collision Avoidance for Airport Traffic Simulation Evaluation, Proceedings of the AIAA/IEEE $29^{\text {th }}$ Digital Avionics Systems Conference. 
[15] Green, D. F, S. D. Otero, G. D. Barker, D. R. Jones, 2009, Initial Concept for Terminal Area Conflict Detection, Alerting, and Resolution Capability On or Near the Airport Surface, NASA TM-2009-215696.

[16] Department of Transportation Federal Aviation Administration, 2010, Aeronautical Information Manual.

[17] Department of Transportation Federal Aviation Administration, 2009, Airport Design, Advisory Circular No. 150/5300-13, Change 15.
[18] Department of Transportation Federal Aviation Administration, 2010, Standards for Airport Markings, Advisory Circular No. 150/5340-1K.

\section{Acknowledgements}

The authors would like to express their appreciation to the Air Traffic Operations Laboratory and Aeronautics Systems Engineering Branch personnel for their support throughout all phases of implementation, testing, and analysis.

31st Digital Avionics Systems Conference

October 14-18, 2012 